\title{
Biosensor on the Basis of Planar Nanostructure with Built-In Enzymatic Molecular Complexes
}

\author{
Kolesov V. V., Krupenin S. V., Soldatov E. S., and Reshetilov A. N.
}

\begin{abstract}
In the work a planar topology for nanoelectronic transducer based on the enzyme glucose oxidase is developed. An experimental study of the possibility of forming nanogaps in planar nanostructures by electromigration is carried out. A technique of implementation of planar nanostructures for nanoelectronic biosensor based on the methods of electron-beam lithography is developed. A technological map of manufacturing of nanogaps between metal electrodes by electromigration of metal atoms on the basis of a computer-controlled process of breaking metal nanowires is developed and realized. The method of the chemical modification of silicon dioxide surface by epoxysilane was developed and the immobilization of the glucose oxidase enzyme on the surface of planar nanostructure for the nanoelectronic transducer through the linker molecules was realized. The registration procedure of the biochemical signals was developed. A change in the functional state of the immobilized ferment of the glucose oxidase enzyme with the presence (oxidation) of glucose in the test solution was demonstrated.
\end{abstract}

Index Terms-Biosenor, nanotransistor, nanostructures, enzymatic electrochemical sensors, enzymatic activity, environmental monitoring.

\section{INTRODUCTION}

The biosensors represent the complex analytical devices that use biological materials for "recognition" of certain molecules and give information about their presence and quantity in the form of an electrical signal. The principle of analysis, realized in biosensors, based on the fact that the biological material (enzymes, cells, organelles, immunocomponents) immobilized on physical sensors, produces at the interaction with the analyzed compounds a concentration-dependent electric signal [1].

Generally the operation of the majority of modern biosensors is based on the enzymatic catalysis. A conjugation of the enzymatic catalytic and electrochemical reactions occurring on conductive materials, immersed in an electrolyte solution, allow to develop a lot of biosensors for the determination of various biologically active compounds.

As one of the most common model of biocatalysts for the biosensor analysis the glucose oxidase and alcohol oxidase

Manuscript received January 3, 2013; revised March 3, 2013. The work was partially supported by the Ministry of Science and Education of Russian Federation, Russian Foundation of Basic Research (Grant \#12-07-00816-a) and President Grant \#MK-6718.212.9.

Kolesov V. V. and Krupenin S. V. are with Institute of Radioengineering \& Electronics, Russian Academy of Sciences, Moscow, Russia (e-mail: kvv@cplire.ru; krupenin@cplire.ru).

Soldatov E. S. is with Faculty of Physics, Moscow State University, Moscow, Russia (e-mail: esold@phys.msu.ru)

Reshetilov A. N. is with Institute of Biochemistry and Physiology of microorganisms, Russian Academy of Sciences, Moscow, Russia (e-mail: anatol@ibpm.pushchino.ru) are used. The glucose oxidase is a highly selective enzyme with in details studied characteristics. The alcohol oxidase from methylotrophic yeast Pichia angusta is capable of catalyzing the oxidation of aliphatic alcohols, and is used in biosensors for analysis [2].

Functionally the biosensors are comparable to sensors of a live organism - the bioreceptors, capable to transform all types of the signals arriving from environment, to the electric ones. Now the most widely biosensors based on enzymes are used. A significant amount of enzymatic biosensors is focused on the analysis of liquids, including biological (for example, for express diagnostics of glucose concentration in blood). Besides, the enzymatic biosensors find application in biotechnology, the food industry, the nature conservation and adjacent areas. They help to solve the various biomedical problems and control the state of the environment (for example, for control of the content of toxic substances) [3].

Any biosensor consists of two basic functional elements: the bioselective element using various biological structures and the physical converter of the signal (transducer) transforming a concentration signal to the electric signal one. The electronic amplification and registration systems are used for reading and recording of the information. As a bioselective element it is possible to use all types of biological structures: enzymes, antibodies, receptors, nucleonic acids and even living cells. In turn the electrochemical converters (electrodes), different optical converters, gravitational, calorimetric, resonant systems can be used as the transducers. All types of bioselective elements can be combined with various transducers. It makes a large variety of various types of biosensors.

As a whole the class of enzymes- oxidases is high-specific in relation to defined substrata. The advantage of this type of a biosensor is first of all in its high selectivity which is defined by specificity of used enzymes and the nature of electrochemical reaction in which the components of enzymatic process participate. On the contrary a systems on the basis of not biological converter, are not selective that is caused by a variety of reasons.

The last achievements in a solution of the problem of miniaturization electronic devices, and also successes in combination of technologies of biology and nanoelectronics allow to develop original versions of nanodevices, in particular, transistors on the basis of nanowires and single molecules that gives an essentially new possibility of use of nanostructures for not only the development of miniature physical devices, but also for the solution of more wide range of tasks in live systems, for example, for production of the miniature biosensor diagnostic units [4].

\section{Manufacturing TechniQues of Planar NANOSTRUCTURE}


To provide an electrical connection with nanostructures, previously the chip infrastructure of the supplying gold wires conducting in the central region of size $90 \times 90$ micrometers was made. The transfer of mask pattern on a substrate was carried out by photolithography. For a photolithography the technology of the double-layer mask (Fig. 1) was used. This technology allows avoiding low-quality border due to contact of sputtering metal with photoresist walls. [5].

The double -layer technology consists of several stages: application of isolating silicon dioxide on a silicon substrate, consecutive application of layers of copolymer and polymer, the UV exposure, processing in toluol and alcohol mixture, a chrome and gold sputtering, mask dissolution in acetone.

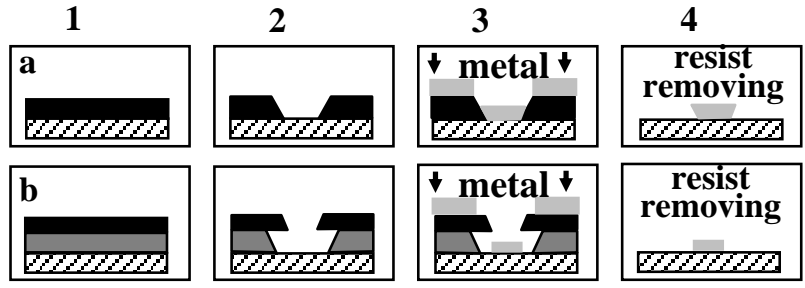

1-drawings a resist, 2- development, 3-puttering of metal and 4- removing of a resist with rest of metal.

Cross-hatched area- $\mathrm{SiO}_{2}$, black area- resist Shipley $\mathrm{S} 1805$, gray area- resist PMGI SF6, light gray area- metal.

Fig. 1. Comparison single-layer (a) and double-layer (b) of lift-off technology.

A resist PMGI SF6 (MicroChem) was used as the bottom layer of the mask, and Shipley S1805 was used as the top resist layer. The photoresist was exposed by means of installation and exposition system SET MA-750. The double-layer resistive mask was developed in MF-319 developer (Metal Free). As a result, the areas, free from the resist (so-called "windows") appear on a substrate in the exposed places after this intermediate stage. The size of the canopy of the top resist over bottom can be regulated by change of drying temperature of the bottom resist layer.

Into the resulting "windows" in the photoresist a buffer layer of chromium ( $2 \mathrm{~nm}$ ) (for adhesion of gold on silicon oxide) and layer of gold (40 nm) were thermally deposited at a pressure of $9 \times 10^{-7}$ mbar by Leybold L-560. As a result, after removing of photoresist the structure of macroelectrodes (Fig. 2) on the chip was formed.

The measuring nanostructure is disposed in the central area of the chip $(90 \times 90$ microns $)$ and it was a prototype of system of electrodes for the molecular transistor. The formation of nanostructure was carried out by high resolution lithography with an electron microscope with energy of about $10 \mathrm{keV}$. In work MicroChem 950 PMMA C2 polymer was used as a top resist layer, and the copolymer MMA(8.5)MAA EL11 was used as the bottom one.

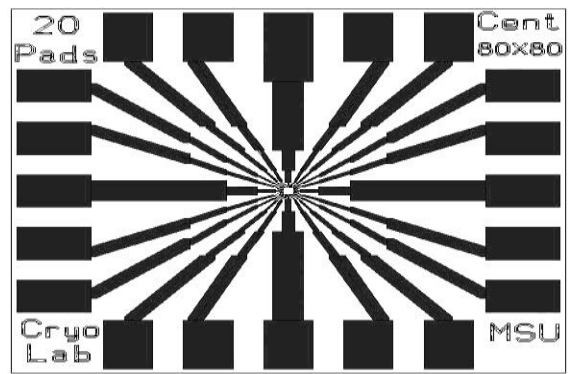

Fig. 2. The topology of supplying electrodes on the chip. Size of chip is $10 \times 10 \mathrm{~mm}^{2}$.
The resulting copolymer film thickness is about $500 \mathrm{~nm}$, and the thickness of the polymer film about $150 \mathrm{~nm}$. The system of measuring electrodes with nanowires in the central part of the chip is shown in Fig. 3.

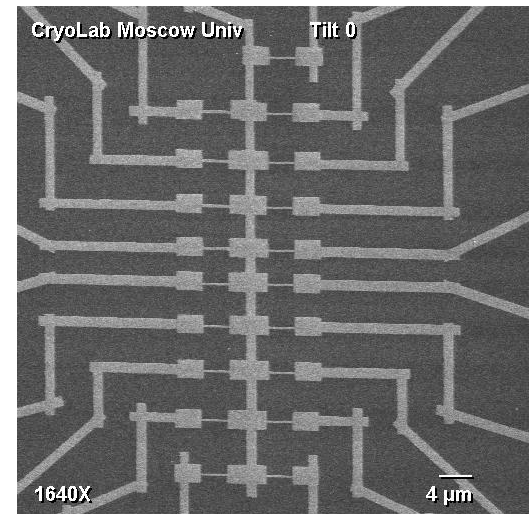

Fig. 3. The nanoelectrodes topology of the nanostructure.

The measuring nanoelectrodes for the molecular transistor can be obtained by a breakup of a gold film with forming a gap of nanometer scale [6]. For a breakup of a thin-film nanowire the electromigration technique can be effectively used. This technique requires the ability to set a high density current through a gold nanowire, and control its parameters in real time. By means of electromigration technology the breakup in the center of a nanowire was made and the technological nanogaps for fixing of enzyme molecules were formed (Fig. 4).

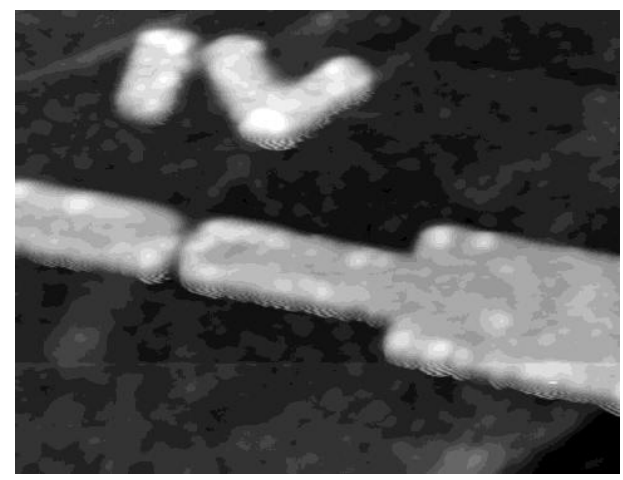

Fig. 4. The nanogap image. Size of image is $2 \times 2 \mu \mathrm{m}^{2}$.

\section{The Technology of An EnZyme of Molecules IMMOBILIZATION}

In the experiments three enzymatic preparations of glucose oxydase (GOD) were used: GOD synthesized of Penicillium adametzii LF F-2044.1 (activity of $41.4 \mathrm{unit} / \mathrm{mg}$ ), GOD of Penicillium funiculosum 46.1 (activity of 36.4 unit $/ \mathrm{mg}$ ), and GOD of Aspergillus niger (activity of 265 unit/mg) (Valeant Pharmaceuticals, the USA). At preliminary research of properties of glucose oxydases there was checked the influence of active acidity in the range of $\mathrm{pH} 2-12(0.01 \mathrm{M}$ the universal buffer) and temperatures in the range of 20-80 ${ }^{\circ} \mathrm{C}$. [7].

For an immobilization of glucose oxidase enzyme on the composite substrate, which was a silicon covered by silicon dioxide, the cross-link method of a protein groups through a epoxysilane groups to a hydroxyl groups on a the surface of a silicon dioxide was applied. 


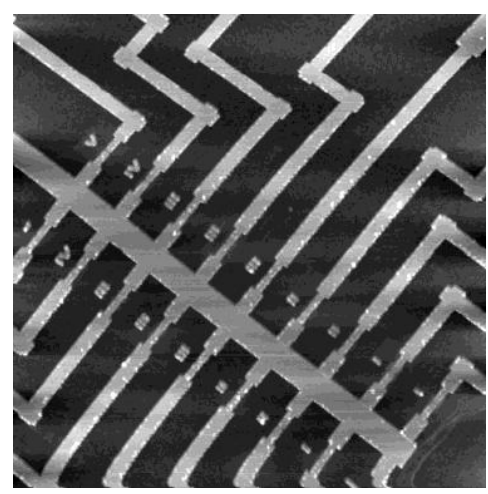

Fig. 5. AFM-image of the nanostructure covered with a molecular layer of a glucose oxidase enzymes. Size of image is $20 \times 20 \mu \mathrm{m}^{2}$.

The produced topological structure was investigated by methods of atomic force microscopy (AFM). In Fig. 5 it is shown the AFM-image of nanoelectrode structure of a transducer with system of operating nanogaps after the modification of surface and putting protein of the glucose oxidase enzyme.

By means of a phase contrast mode (Phase Imaging) based on a base of methods of contactless atomic force microscopy the areas of nanogaps are investigated. The method of phase contrast allows to investigate with high spatial resolution a heterogeneity of various properties on a surface of materials. In particular, the method of phase contrast allows to distinguish the areas differing on a chemical composition, adhesive and elastic properties. The principle of action is based on detecting of a phases difference between mechanical oscillations of a probe near a surface of a sample and the electric signal which excites these oscillations by piezodrive. On the phase contrast images of a surface of gold nanoelectrodes it is visible the separate "soft" aggregated objects which elastic properties are different from the rest of the surface. On the image it is visible that the part of molecular aggregates is fixed inside of a nanogap and included in an electric circuit of the transducer (Fig. 6).

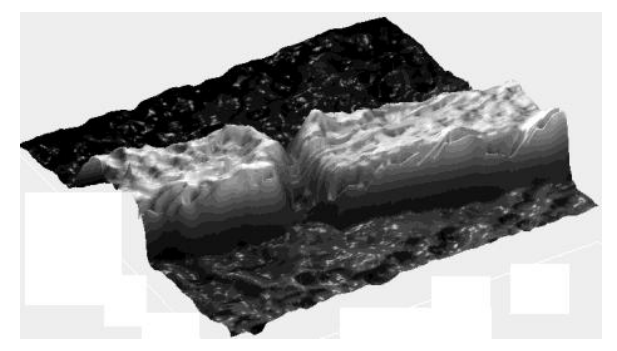

Fig. 6. AFM -image of a separate nanogap with glucose oxidase enzymes. Size of image is $1 \times 1 \times 0.8 \mu \mathrm{m}^{3}$.

\section{THE RESEARCH OF ELECTRONIC TRANSPORT PROCESSES IN NANOSTRUCTURES WITH AN ENZYMATIC COMPLEX}

The studied model of a nanoelectronic transducer represented planar nanostructure from the 18 separate operating nanogaps formed on the silicon chip of 9x9 mm in size by means of methods of electronic lithography and electromigration. The chip is supported on a chipholder and it was electrically connected by an aluminum wire with a diameter of 25 microns to measuring external contacts by means of technology of ultrasonic bonding (Fig. 7).
The research of the model of a nanoelectronic transducer on the basis of planar nanostructure with built-in molecules of enzymes was preceded by preliminary experiments with use of converters of electrochemical type. There were used the substrates with gold nanoelectrodes. There was investigated the connection of electrodes to glucose oxidase enzymes which deposited in the form of solution, and also immobilized with use of glutaraldehyde (an enzyme put in microquantities). The obtained results were compared further to data on electronic transport processes of the transfer of a charge in nanostructures.

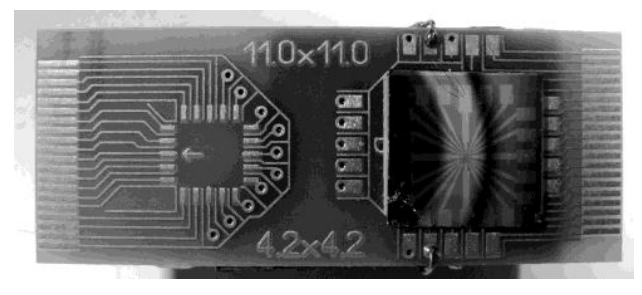

Fig. 7. The model of a nanoelectronic transducer: the silicon chip with the nanoelectronic structure, mounted on a chipholder. Size of the chipholder is $4 \times 2 \mathrm{sm}^{2}$.

The electronic properties of a nanoelectronic transducer were investigated in buffer solution both in absence and at addition of a glucose oxidase. It is shown that reaction to glucose is absent on a control sample (Fig. 8), and an electronic response of the biosensor to $10 \mathrm{MM}$ solution of glucose is present on the nanoelectronic structure modified by enzyme (Fig. 9).

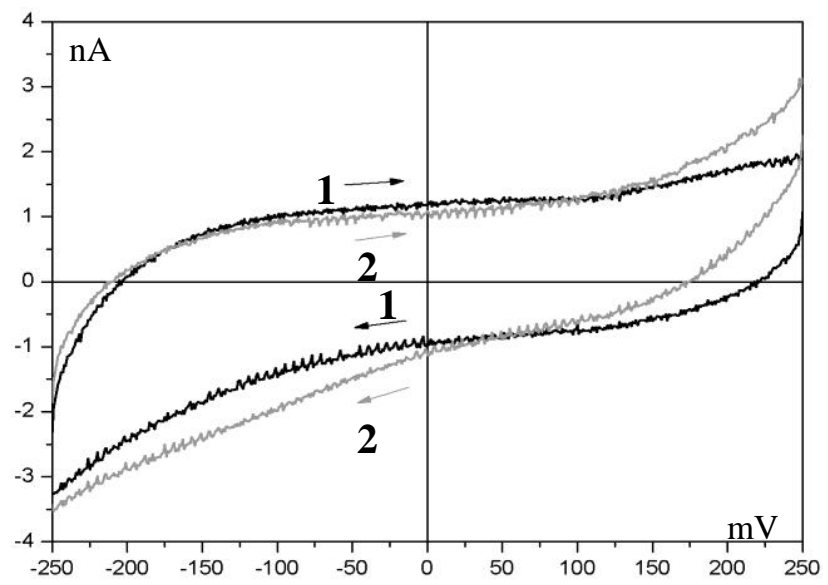

Fig. 8. The test sample without GOD. The reaction on a glucose is absent. 1water, 2- glucose $10 \mathrm{mM}$.

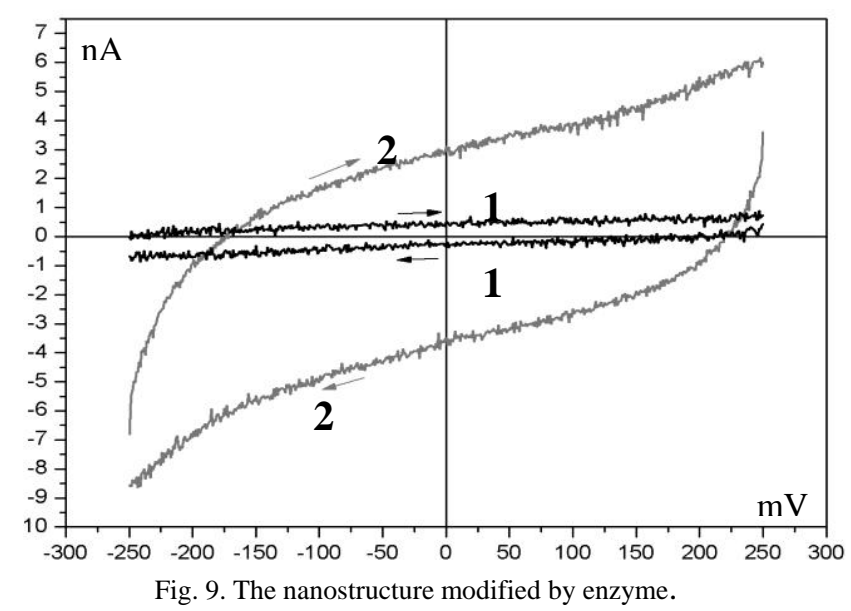

There is an electronic response of the sensor to $10 \mathrm{MM}$ glucose solution. 1 buffer, 2 - buffer + glucose $10 \mathrm{mM}$. 


\section{CONCLUSION}

Thus, in work the production technology of the model of the nanoelectronic transducer on the basis of planar nanostructure with built-in enzymatic complexes is designed. The technology of an immobilization of molecules of enzyme on nanostructure electrodes is elaborated. The technique of biochemical signals recording is developed. The change of a functional state of the immobilized enzyme of glucose oxidase in the presence of glucose (oxidation) in the test solution is demonstrated.

\section{ACKNOWLEDGMENT}

The work was partially supported by the Ministry of Science and Education of Russian Federation, Russian Foundation of Basic Research (Grant \#12-07-00816-a) and President Grant \#MK-6718.212.9.

\section{REFERENCES}

[1] M. Campàs and I. Katakis, Trends Anal Chem, vol. 23, no. 1, pp. 49-62, 2004.

[2] G. E. D. Benedetto, F. Palmisano, P. G. Zambonin, "One-step fabrication of a bienzyme glucose sensor based on glucose oxidase and peroxidase immobilized onto a poly(pyrrole) modified glassy carbon electrode," Biosensors and Bioelectronics, vol. 11, no. 10, pp. 1001-1008, 1996.
[3] H. Harms, M. C. Wells, J. R., and V. D. Meer, Appl Microbiol Biotechnol, vol. 70, pp. 273-280, 2006.

[4] S. P. Gubin, Y. V. Gulyaev, G. B. Khomutov, V. V. Kislov, V. V. Kolesov, I. A. Maximov, L. Samuelson, E. S. Soldatov, and I. V. Taranov, "Electronics of molecular nanoclusters," International Journal of Nanoscience, vol. 3, no. 1-2, 2004, pp. 137-147.

[5] V. V. Kolesov, I. V. Sapkov, and E. S. Soldatov, "Molecular single electron transistor on the basis of the planar nanostructure," in Proc. 20-th Int. Crimean Conference "Microwave \& Telecommunication Technology" (Crimico'2010), 13-17 September 2010, Sevastopol, Crimea, Ukraine, Proceedings, pp. 857-858.

[6] V. V. Kolesov, V. Sapkov, and E. S. Soldatov, "Using a Focused Ion Beam for the Creation of a Molecular Single Electron Transistor," Moscow University Physics Bulletin, vol. 64, no. 4, pp. 384-388, 2009.

[7] H. U. Bergmeyer, K. Gawehn, and M. Grassl, Methods of Enzymatic Analysis, Ed. Bergmeyer H.U. New York: Academic Press, Inc. 1974, 2 ed. vol. 1. pp. 457-458.

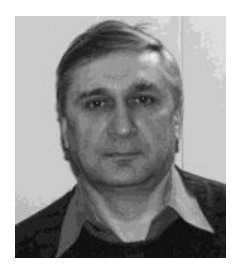

Vladimir Vladimirovich Kolesov graduated from the Physical Department of the MSU in1974. He received his Ph.D. in physical and mathematical science in 1985 at Physical Department of the MSU. $\mathrm{He}$ is currently a head of laboratory at Kotel'nikov IRE RAS. His main research interests include the molecular electronics, nanotechnology, nonlinear oscillations and waves, dynamical systems and chaos, signal processing, biomedical electronics, information technology. His research has been reported in more than 100 articles in international and Russian scientific journal and five inventions. 